\title{
Clinical Profile of Seizure Disorder in Patients Hospitalised in Kanyakumari Government Medical College
}

\author{
Authors \\ Dr Anny Antony ${ }^{1 *}$, Dr Jenix Nathan.Y.A ${ }^{2}$ \\ ${ }^{1}$ Assistant Professor, Dept. of General Medicine, Kanyakumari Government Medical College \\ ${ }^{2}$ Junior Resident, Dept. of General Medicine, Kanyakumari Government Medical College \\ *Corresponding Author \\ Dr Anny Antony \\ Assistant Professor, Dept. of General Medicine, Kanyakumari Government Medical College
}

\section{Introduction}

The word "epilepsy" is derived from Latin and Greek words for "seizure" or "to seize upon". The periodicity of seizures are often worrisome and bothersome that has got a social impact which includes fear, segregation, isolation which inturn affect the self esteem of the patient and hasa deteriorating effect on patient and his family. Epilepsy is most common disorder in kanyakumari medical college. In past epilepsy is thought as possesion of evil spirit. In kanyakumari there are times were people will be taken for temples, mosque and churches for seizure attack. Despite latest advances epilepsy remains a social problem. Widespread ignorance, fear, misunderstanding and stigma contributed to severe legal and social penalties

\section{Need For Study}

Knowledge regarding people affected with epilepsy is essential for accurate planning and management of patients.

\section{Aim}

To study clinical profile of seizure disorder in patients hospitalized in kanyakumari government medical college.

\section{Materials and Methods}

This study is a single centre hospital based cross sectional study of clinical profile of seizure disorder in patients hospitalized in kanyakumari government medical college. Inclusion criteria being all patients presenting with complaints of seizures.

\section{Methodology}

This study included a total of 107 patients which includes 60 male patients and 47 female patients. All patients were included in study after a written informed consent. And they were subjected to a detailed History taking and clinical examination by our residents and detailed analysis was done AGE distribution

\begin{tabular}{|l|c|}
\hline Age & Number of patients \\
\hline $10-20$ & 8 \\
\hline $20-30$ & 7 \\
\hline $30-40$ & 11 \\
\hline
\end{tabular}




\begin{tabular}{|l|c|}
\hline $40-50$ & 14 \\
\hline $50-60$ & 21 \\
\hline $60-70$ & 18 \\
\hline $70-80$ & 14 \\
\hline $80-90$ & 10 \\
\hline $90-100$ & 4 \\
\hline
\end{tabular}

A total of 107 patients were included in the study out of which 8 patients were from 10-20 years of age group, 7 patients were from 20-30 years of age group, 11 patients were from 30-40 years of age group, 14 patients were from $40-50$ years of age group, 21 patients were from 50-60 years of age group, 18 patients were from $60-70$ years of age group, 14 patients were from 70-80 years of age group, 10 patients were from $80-90$ years of age group, 4 patients were from 90-100 years of age group. Maximum number of patients were in $50-60$ years and least number of patients were in 90-100 years

\section{Sex}

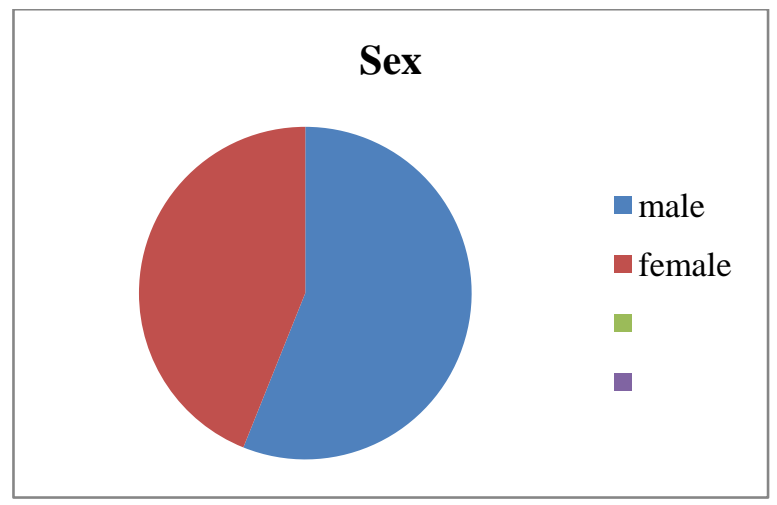

A total of 107 patients which includes 60 male patients and 47 female patients. Even though there is no overall gender difference in seizure disorder. In our study seizure disorder was more in males.

\section{Smoking and Alcohol}

Smoking and alcohol increases the seizure thresold. so on withdrawal seizure may occur.

In our study the following chart indicates percentage of smokers, alcoholic and non alcoholic

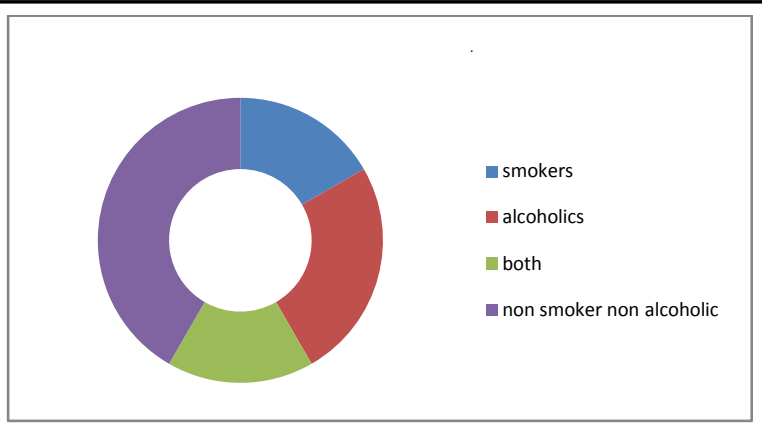

\section{Last Seizure Episode}

$30 \%$ of patients had new onset seizures.20\% patients had seizure 6 months back. $15 \%$ had last seizure episode 1 year back. Few didn't remember there last seizure episode. $4 \%$ patients had seizure episode within one week.

\section{Seizure Provocative Factor}

In most of the cases seizure occurs spontaneously, but there may be association with various triggers. These triggers may act as seizure precipitating factors .All participants were interviewed through a predesigned close ended questionnaire that included a long list of 20 precipitating factors. Fever precipitated seizure in $16 \%$ of the patients. $17 \%$ noticed that whenever there was sleep deprivation, they had an attack of epilepsy. However, drug withdrawal, playing outside are provocative factors. Most of the patients can identify their seizure precipitant and clustering of many SPFs suggests a common patho-physiologic mechanism for these triggers. Excitation, sleep deprivation, fever, watching television and head trauma showed a strong association with generalized epilepsy. Patients with seizure disorder should be evaluated for presence of SPFs, because identification of these might help in proper management of epilepsy. 


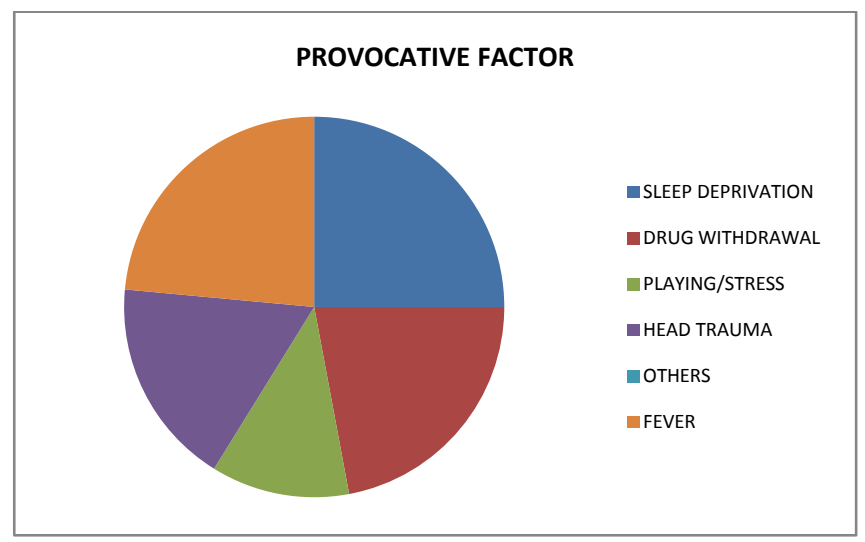

\section{Demography}

In our study population $75 \%$ of patients fall under low socioeconomic group and $73 \%$ of people are uneducated. Hence uneducation and low socioeconomy make people vulnerable to seizures because of lack of awareness, poor compliance to medicines, not detection of seizure provoking factor, repeated exposure to seizure provoking factor, prevalence of alcoholism and thus lowering seizure threshold etc.

\section{Types of Seizures}

Seizures were broadly classified in to 3 types Focal seizures, Generalized seizures and lastly unclear epileptic spasm. Generalised seizures were again classified in to 5 types they are 1.absence seizures, 2.Tonic-clonic seizure, 3.clonic, 4. Atonic and 5.Myoclonic seizure.

Among total of 107 patients in our study 15 patients had focal seizures, 70 patients had generalised seizures and others were unclear epileptic spasm.

\section{Past Medical Illness}

Many medical illnesses can cause seizures. Metabolic derangements, such as disorders of serum glucose metabolism, cause seizures, as well as other neurologic manifestations. In our study out of 107 patients 53 patients were diabetics among diabetics 31 were male and 22 were female, there were 47 hypertensive patients out of which 27 were females and 20 were males. There was about 24 CKD patients out of which 7 patients were on haemodialysis. 4 diabetic patients came with hyperglycemia and focal seizures. 12 patients Had history of RTA. 15 patients had CVD.2 patients had developmental delay.

\section{Clinical Profile}

Almost all patients had postictal confusion. Maximum duration of post ictal state was about 24 hours and minimum duration of post ictal state was about 30 minutes. 5 patients had slurring of speech. 2 patients had neck stiffness. Fecal incontinence in 5 patients. 15 patients had focal neurological deficit. 6 patients have bitten there tongue.

\section{Response to Treatment}

98\% of patients responded to treatment and they didn't had recurrent episodes of seizure. $2 \%$ had refractory seizures. The sample size of our cohorts is relatively small. It is possible that some prognosis factors may be missed due to the small sample size. Further studies with a larger sample cohort are required.

\section{Brain Imaging}

Due to lack of resources imaging was not done in all patients. CT brain done in 64 patients. And MRI brain done in 20 patients. 45 patients had normal study. 20 patients had acute or chronic infarct. Brain oedema was found in 19 patients.

\section{EEG}

22 patients had abnormal epileptiform activity in EEG.35 patients had normal EEG study. Few patients missed EEG.

\section{Diagnosis}

\begin{tabular}{|l|c|}
\hline Diagnosis & Percentage \\
\hline Stroke & 25 \\
\hline Medication change & 16 \\
\hline Alcohol & 15 \\
\hline Hypoxia & 11 \\
\hline Metabolic & 12 \\
\hline Unknown & 14 \\
\hline Infection & 4 \\
\hline Tumors & 3 \\
\hline
\end{tabular}

Maximum number of patients presenting with seizure being diagnosed as stroke and tumors being least cause for seizure. 


\section{Conclusion}

The sample size of our cohorts is relatively small. It is possible that some prognosis factors may be missed due to the small sample size. Further studies with a larger sample cohort are required. Uneducation and low socioeconomy make people vulnerable to seizures because of lack of awareness, poor compliance to medicines, not detection of seizure provoking factor, repeated exposure to seizure provoking factor, prevalence of alcoholism and thus lowering seizure threshold etc. there is a treatment gap still because of epilepsy and appropriate usage of resources will help to reduce this treatment gap and decreases epilepsy associated morbidity and mortality.

\section{Disclosure}

The author reports no conflict of interest in this study.

\section{References}

1. Harrisons, Principles of Internal Medicine

2. Sandhya A Kamath API Text book of Medicine

3. CHANGB, LOWENSTEIN D: Mechanisms of disease: Epilepsy. N Engl J Med349:1257, 2003

4. LOWENSTEINDH, ALLDREDGE BK: Status epilepticus. N Engl J Med 338:970, 1998

5. NOEBELSJ: Exploring new gene discoveries in idiopathic generalized epilepsy.Epilepsia 44(Suppl 2):16, 2003

6. PACKAM, MORRELL MJ: Treatment of women with epilepsy. SeminNeuro22:289, 2002

7. WIEBES et al: A randomized, controlled trial of surgery for temporal-lobeepilepsy. N Engl J Med 345:311, 2001. 